STRUCTURAL BIOLOGY COMMUNICATIONS

ISSN 2053-230X

\section{Crystal structure of the Schizosaccharomyces pombe U7BR E2-binding region in complex with Ubc7}

\author{
Zachary S. Hann, ${ }^{\text {a,b }}$ Meredith B. Metzger, ${ }^{c}$ Allan M. Weissman ${ }^{c}$ and \\ Christopher D. Lima ${ }^{\mathrm{a}, \mathrm{d}}$ *
}

Received 16 May 2019

Accepted 8 July 2019

Edited by G. G. Privé, University of Toronto, Canada

Keywords: endoplasmic reticulum; ERAD; ubiquitin; protein degradation; ubiquitin-conjugating enzyme; E2; E2-binding protein; Schizosaccharomyces pombe.

PDB reference: Ubc7-U7BR complex, 6op8

Supporting information: this article has supporting information at journals.iucr.org/f

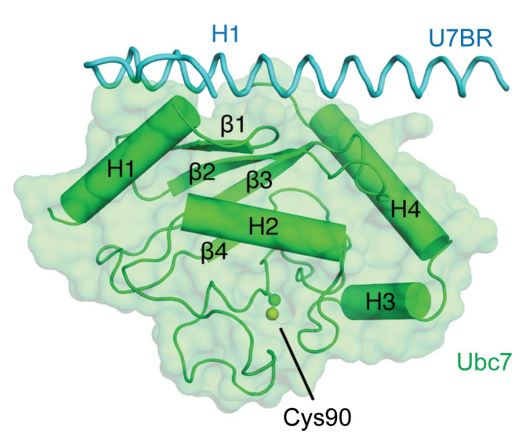

OPEN $\odot$ ACCESS

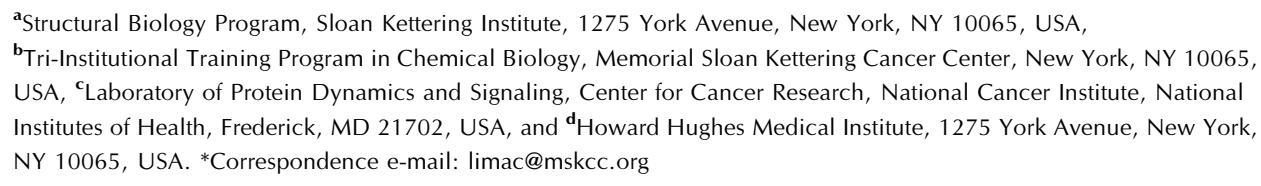

Endoplasmic reticulum (ER)-associated degradation (ERAD) is a protein quality-control pathway in eukaryotes in which misfolded ER proteins are polyubiquitylated, extracted and ultimately degraded by the proteasome. This process involves ER membrane-embedded ubiquitin E2 and E3 enzymes, as well as a soluble E2 enzyme (Ubc7 in Saccharomyces cerevisiae and UBE2G2 in mammals). E2-binding regions (E2BRs) that recruit these soluble ERAD E2s to the ER have been identified in humans and $S$. cerevisiae, and structures of E2-E2BR complexes from both species have been determined. In addition to sequence and structural differences between the human and $S$. cerevisiae E2BRs, the binding of E2BRs also elicits different biochemical outcomes with respect to E2 charging by E1 and E2 discharge. Here, the Schizosaccharomyces pombe E2BR was identified and purified with Ubc7 to resolve a $1.7 \AA$ resolution co-crystal structure of the E2BR in complex with Ubc7. The S. pombe E2BR binds to the back side of the E2 as an $\alpha$-helix and, while differences exist, it exhibits greater similarity to the human E2BR. Structure-based sequence alignments reveal differences and conserved elements among these species. Structural comparisons and biochemistry reveal that the $S$. pombe E2BR presents a steric impediment to E1 binding and inhibits E1-mediated charging, respectively.

\section{Introduction}

Eukaryotic membrane and secreted proteins are translated on and folded in the endoplasmic reticulum (ER; Smith et al., 2011). Misfolding of this class of proteins elicits ER stress and can affect cellular homeostasis. The accumulation of such misfolded proteins and ER stress underlies many human disorders (Guerriero \& Brodsky, 2012). Cells protect against this through pathways that include ER-associated degradation (ERAD), a process in which incorrectly folded proteins are ubiquitylated, marking them for extraction and subsequent degradation by the proteasome (Zattas \& Hochstrasser, 2015).

ERAD requires specific E2 and E3 enzymes, including a conserved soluble E2: UBE2G2 (humans) or Ubc7 (Saccharomyces cerevisiae) (Smith et al., 2011; Ye \& Rape, 2009; Hiller et al., 1996; Biederer et al., 1996; Tiwari \& Weissman, 2001). These E2s are recruited to the ER by membrane-embedded proteins. In humans, this is accomplished by gp78, a polytopic E3 in which the cytoplasmic tail includes a RING domain that confers E3 activity, a CUE domain that binds Ub noncovalently (Biederer et al., 1997; Prag et al., 2003) and a C-terminal 
27-residue UBE2G2-binding region (G2BR; Chen et al., 2006). X-ray crystallographic studies of the UBE2G2-G2BR complex show G2BR to bind to the back side of the E2 (the face opposite the catalytic cysteine) as an $\alpha$-helix (Li et al., 2009; Das et al., 2009). In isolation, G2BR decreases the rate of UBE2G2 Ub charging by the Ub-activating E1 enzyme but increases the rate of RING domain-dependent UBE2G2 Ub discharge to substrates (Das et al., 2009).

In $S$. cerevisiae, Ubc7 is recruited to the ER by Cue1, a single-pass transmembrane protein that includes a transmembrane helix, a CUE domain and a Ubc7-binding region (U7BR) composed of 53 residues at its C-terminal end (Kostova et al., 2009). Unlike gp78 in humans, S. cerevisiae Cue1 does not possess a RING domain or catalyze E3 activity, but it enhances the rate of Lys48-linked Ub chain building on ERAD substrates (Bazirgan \& Hampton, 2008; Kostova et al., 2009) by pairing with one of two ER-embedded RING domain-containing Ub E3s: Hrd1 and Doa10 (Biederer et al., 1997; Ravid et al., 2006; Swanson et al., 2001). The U7BR domain is necessary for the degradation of Ubc7 substrates, presumably because it recruits Ubc7 to the ER (Kostova et al., 2009). Unlike human G2BR, Cue1 and its U7BR domain increase the rate of E1-mediated E2 charging (Metzger et al., 2013).

Human and S. cerevisiae E2BRs both bind to the back side of their respective E2s through $\alpha$-helices, but share less than $65 \%$ sequence similarity, and only two E2-E2BR hydrogenbond contacts appear to be conserved in their structures. In addition, while human G2BR is composed of a single long $\alpha$-helix, S. cerevisiae U7BR consists of a shorter $\alpha$-helix that is flanked by two $\alpha$-helices (H1 and $\mathrm{H} 3$ ) and a $3_{10}$-helix (g1) (Li et al., 2009; Das et al., 2009; Metzger et al., 2013).

The differences between the human and $S$. cerevisiae E2/E2BR systems suggest that the analysis of other ERAD E2/E2BRs could reveal additional differences that contribute to E2 binding and recruitment in other organisms. Here, we identify the putative E2BR domain in $S$. pombe Cue4 and present a $1.7 \AA$ resolution co-crystal structure of this E2BR in complex with $S$. pombe Ubc7. Structure-based comparisons with human and S. cerevisiae E2BRs reveal overall similarities in addition to structural features that are distinct from either Homo sapiens G2BR or S. cerevisiae U7BR. Similar to human G2BR, S. pombe U7BR may inhibit E1-mediated E2 charging.

\section{Materials and methods}

\subsection{Cloning}

The $S$. pombe Ubc7 gene was inserted into the pET-29b+ vector using the NdeI and XhoI restriction sites. A thrombin cleavage consensus site (LVPRGS) was inserted between the C-terminus of Ubc7 and the C-terminal $\mathrm{His}_{6}$ tag. Residues 152-215 of $S$. pombe Cue4 (U7BR) were inserted into the pET-28b vector using the NdeI and XhoI restriction sites, yielding a construct with an $\mathrm{N}$-terminal thrombin-cleavable $\mathrm{His}_{6}$ tag.
Table 1

Crystallization.

\begin{tabular}{ll}
\hline Method & Hanging drop \\
Plate type & VDXm plate with sealant \\
Temperature (K) & 291 \\
Protein concentration $\left(\mathrm{mg} \mathrm{ml}^{-1}\right)$ & 6.6 \\
Buffer composition of protein & $20 \mathrm{~m} M$ Tris pH $8.0,50 \mathrm{~m} M \mathrm{NaCl}, 1 \mathrm{~m} M$ \\
$\quad$ solution & $\mathrm{TCEP}$ \\
Composition of reservoir solution & $100 \mathrm{~m} M$ Tris pH $8.0,200 \mathrm{~m} M \mathrm{MgCl}_{2}$, \\
& $30 \%$ PEG 4000 \\
Volume and ratio of drop & $1 \mu \mathrm{l}, 1: 1$ \\
Volume of reservoir $(\mu \mathrm{l})$ & 500 \\
\hline
\end{tabular}

\subsection{Protein expression and purification}

$S$. pombe Ubc7 and U7BR were expressed in E. coli BL21-CodonPlus (DE3)-RIL cells. The cells were grown in SuperBroth at $37^{\circ} \mathrm{C}$ to an $\mathrm{OD}_{600}$ of 1.0. IPTG was added to $1 \mathrm{~m} M$ and the temperature was reduced to $30^{\circ} \mathrm{C} .4 \mathrm{~h}$ after induction, the cells were centrifuged for $12 \mathrm{~min}$ at $4^{\circ} \mathrm{C}$ and $5000 \mathrm{~g}$, suspended in buffer consisting of $20 \mathrm{mM}$ Tris $\mathrm{pH} 8$, $350 \mathrm{mM} \mathrm{NaCl}, 20 \%$ sucrose, flash-frozen in liquid nitrogen and stored at $-80^{\circ} \mathrm{C}$ until lysis. Cells were lysed by sonication and cleared by centrifugation at $40000 \mathrm{~g}$ and $4^{\circ} \mathrm{C}$ for $30 \mathrm{~min}$. The cleared lysate was applied onto Ni-NTA Superflow resin (Qiagen), washed in $20 \mathrm{~m} M$ Tris $\mathrm{pH} 8.0,350 \mathrm{~m} M \mathrm{NaCl}, 1 \mathrm{~m} M$ $\beta$-mercaptoethanol, $20 \mathrm{~m} M$ imidazole and eluted in the same buffer with $250 \mathrm{~m} M$ imidazole.

\subsection{Preparation of the Ubc7-U7BR complex}

A solution containing $S$. pombe Ubc7 and U7BR each at $16 \mu M$ was incubated for $80 \mathrm{~min}$ on ice in a buffer consisting of $20 \mathrm{~m} M$ Tris $\mathrm{pH}$ 8.0, $50 \mathrm{~m} M \mathrm{NaCl}, 1 \mathrm{~m} M$ TCEP. The solution was subjected to size-exclusion chromatography on a HiLoad 26/600 Superdex 75 prep-grade column (GE) equilibrated in $20 \mathrm{~m} M$ Tris pH 8.0, $50 \mathrm{~m} M \mathrm{NaCl}, 1 \mathrm{~m} M$ TCEP. Fractions containing the Ubc7-U7BR complex were pooled, concentrated, flash-frozen in liquid nitrogen and stored at $-80^{\circ} \mathrm{C}$ until further use.

\subsection{Crystallization and data collection}

The $S$. pombe Ubc7-U7BR complex was subjected to sparse-matrix crystallization screening using the sitting-drop vapor-diffusion method with a Mosquito robot $(100 \mathrm{nl}$ well solution added to $100 \mathrm{nl}$ protein solution). The Classics Suite and the PEGs Suite from Qiagen were tested at proteincomplex concentrations of $3.3,6.6$ and $10 \mathrm{mg} \mathrm{m}^{-1}$ and temperatures of 4 and $18^{\circ} \mathrm{C}$. An initial crystallization hit appeared within $24 \mathrm{~h}$ using $6.6 \mathrm{mg} \mathrm{ml}^{-1} \mathrm{Ubc7-U7BR,} 100 \mathrm{mM}$ Tris $\mathrm{pH} 8.5,200 \mathrm{mM} \mathrm{MgCl}, 30 \%$ PEG 4000 at $18^{\circ} \mathrm{C}$. Refinement resulted in crystallization conditions that consisted of $100 \mathrm{~m} M$ Tris $\mathrm{pH}$ 8.0, $200 \mathrm{~m} M \mathrm{MgCl}_{2}, 30 \%$ PEG 4000 (Table 1). $1 \mu \mathrm{l}$ of this well solution was added to $1 \mu \mathrm{l}$ protein solution composed of $6.6 \mathrm{mg} \mathrm{ml}^{-1}$ Ubc7-U7BR, $20 \mathrm{~m} M$ Tris $\mathrm{pH} 8.0$, $50 \mathrm{~m} M \mathrm{NaCl}, 1 \mathrm{~m} M$ TCEP on a cover slide and set over $500 \mu \mathrm{l}$ well solution in a hanging-drop vapor-diffusion experiment. The initial crystals diffracted to $2.9 \AA$ resolution; although $\alpha$-helical density was observed on the back side of Ubc7, the maps were of insufficient quality to unambiguously determine 
Table 2

Data collection.

Statistics were calculated using PHENIX; values in parentheses are for the outer shell.

\begin{tabular}{|c|c|}
\hline Diffraction source & 24-ID-C, APS \\
\hline Wavelength $(\AA)$ & 0.97910 \\
\hline Temperature (K) & 100 \\
\hline Detector & PILATUS 6M-F \\
\hline Crystal-to-detector distance (mm) & 250 \\
\hline Rotation range per image $\left({ }^{\circ}\right)$ & 0.2 \\
\hline Total rotation range $\left({ }^{\circ}\right)$ & 180 \\
\hline Exposure time per image (s) & 0.2 \\
\hline Space group & $P 2_{1} 2_{1} 2_{1}$ \\
\hline$a, b, c(\AA)$ & $26.72,79.05,94.52$ \\
\hline$\alpha, \beta, \gamma\left(^{\circ}\right)$ & $90,90,90$ \\
\hline Resolution range $(\AA)$ & $47.26-1.703$ (1.764-1.703) \\
\hline Completeness (\%) & $99.39(98.12)$ \\
\hline Total No. of reflections & $129954(12467)$ \\
\hline No. of unique reflections & $22673(2195)$ \\
\hline Wilson $B$ factor $\left(\AA^{2}\right)$ & 14.49 \\
\hline Multiplicity & $5.7(5.7)$ \\
\hline$R_{\text {merge }}(\%)$ & $5.62(22.32)$ \\
\hline $\mathrm{CC}_{1 / 2}(\%)$ & $99.9(97)$ \\
\hline $\mathrm{CC}^{*}(\%)$ & $100(99.2)$ \\
\hline$\langle I / \sigma(I)\rangle\rangle^{\prime}$ & $25.94(9.05)$ \\
\hline
\end{tabular}

the register of U7BR. The single crystal used to collect the reported data was harvested from a crystallization experiment at $18^{\circ} \mathrm{C}$ after approximately one year. The crystal was cryoprotected by stepwise transfer to drops containing mother liquor plus increasing concentrations of MPD up to a final concentration of $20 \%$ before being flash-cooled in liquid nitrogen. Data were collected on the NE-CAT beamline 24-ID-C at the Advanced Photon Source (APS), Argonne, Illinois, USA (Table 2).

\subsection{Structure determination and refinement}

The data set was indexed, integrated and scaled using $H K L$ 2000 (Otwinowski \& Minor, 1997). Data were truncated at $1.7 \AA$ resolution to maintain completeness as the crystal-todetector distance was not adjusted to obtain higher resolution data for this crystal. Phaser (McCoy et al., 2007) was used for molecular replacement using Ubc7 from the $S$. cerevisiae Ubc7-U7BR structure (PDB entry 4jqu; Metzger et al., 2013) as a search model (with the U7BR chain deleted). The initial solution revealed electron density consistent with a helix near the back side of Ubc7 in a similar position to that observed for the human and $S$. cerevisiae ERAD E2BRs. A polyalanine model was initially placed into this density and refinement resulted in density characteristic of amino-acid side chains. The sequence of $S$. pombe Cue4 was built into this density. Coordinates were refined via iterative rounds of refinement and rebuilding using PHENIX (Adams et al., 2010) and Coot (Emsley et al., 2010) (Table 3).

\subsection{Transthiolation assay}

S. pombe $\mathrm{Uba}^{\Delta 1-12}$ and $\mathrm{Ub}$ were purified as described previously (Olsen \& Lima, 2013). Reactions contained $10 \mathrm{n} M$ Uba1, $500 \mathrm{n} M$ Ubc7, $3 \mu M \mathrm{Ub}, 0$ or $1 \mu M$ U7BR, $10 \mathrm{~m} M$ $\mathrm{MgCl}_{2}, 20 \mathrm{~m} M$ HEPES pH 7.5, $50 \mathrm{~m} M \mathrm{NaCl}, 0.1 \%$ Tween-20. $2 \mathrm{~m} M$ ATP was used to start the reaction. Reactions were run
Table 3

Structure refinement.

Statistics were calculated using PHENIX; values in parentheses are for the outer shell.

\begin{tabular}{ll}
\hline Resolution range $(\AA)$ & $47.26-1.703(1.764-1.703)$ \\
$\sigma$ Cutoff & $F>1.380 \sigma(F)$ \\
No. of reflections (work/free) & $22633(2187) / 1169(98)$ \\
$R_{\text {work }} / R_{\text {free }}(\%)$ & $16.80(19.69) / 19.76(24.65)$ \\
No. of non-H atoms & \\
$\quad$ Total & 1952 \\
$\quad$ Protein & 1732 \\
$\quad$ Ligand & 6 \\
$\quad$ Solvent & 214 \\
R.m.s. deviations & \\
$\quad$ Bonds $(\AA)$ & 0.010 \\
$\quad$ Angles $\left({ }^{\circ}\right)$ & 1.04 \\
Average $B$ factors $\left(\AA^{2}\right)$ & \\
$\quad$ Overall & 20.50 \\
$\quad$ Protein & 19.54 \\
Ligand & 34.27 \\
$\quad$ Water & 27.88 \\
Statistics from MolProbity & \\
$\quad$ Ramachandran favored $\dagger(\%)$ & $97.09[200]$ \\
Ramachandran outliers $\dagger(\%)$ & $0[0]$ \\
Clashscore & 96 th percentile \\
MolProbity score & 91 st percentile \\
PDB code & $60 p 8$ \\
\hline
\end{tabular}

$\dagger$ The number of residues is given in brackets.

at room temperature and quenched after the indicated time by mixing the reaction with an equal volume of $4 \times$ LDS NuPAGE loading dye (Life Technologies). Lanes labeled ' $+\beta \mathrm{ME}$ ' in Supplementary Fig. S1 contained $358 \mathrm{mM}$ $\beta$-mercaptoethanol after mixing the sample and dye. Reactions were resolved by $4-12 \%$ Bis-Tris SDS-PAGE with MOPS running buffer (Life Technologies), stained with SYPRO Ruby (Bio-Rad) and imaged on a Typhoon FLA 9500. Ubc7 bands were quantified using ImageJ (NIH). Each data point in Fig. 4(a) is the average of three independent experiments, and error bars represent one standard deviation. Values were normalized to the percentage of E2-SH remaining by dividing the values by the average value at the zero time point and multiplying by 100 .

\section{Results and discussion}

3.1. Identification, preparation and crystallization of the S. pombe Ubc7-U7BR complex

Protein sequences for $S$. pombe Cue4 U7BR were aligned and domain boundaries were estimated based on the known structure of $S$. cerevisiae U7BR in complex with Ubc7. Alignments between residues 152-215 of S. pombe Cue4 and residues $151-203$ of $S$. cerevisiae Cue1 revealed that the two sequences share $66 \%$ similarity and $38 \%$ identity within the predicted E2-binding surface. The E2 proteins are better conserved than their respective E2BRs, with the $S$. pombe and S. cerevisiae Ubc7 sequences sharing $75 \%$ similarity and $61 \%$ identity. For comparison, the sequences of $S$. pombe U7BR and $H$. sapiens G2BR (residues $579-600$ of gp78) share $70 \%$ similarity and $47 \%$ identity, while the sequences of $S$. pombe 
Ubc7 and human UBE2G2 share $83 \%$ similarity and $68 \%$ identity.

Full-length $S$. pombe Ubc7 and U7BR encompassing residues 152-215 of Cue4 were expressed in E. coli and purified. $S$. pombe Ubc7 and U7BR were incubated in a stoichiometric ratio and purified by size-exclusion chromatography. The peak was pooled, concentrated and subjected to sparse-matrix crystallization screening. After refining the crystallization conditions, a crystal was grown that diffracted to $1.7 \AA$ resolution in space group $P 22_{1} 2_{1} 2_{1}$ with a single complex in the asymmetric unit (Tables 1 and 2). Phases were determined by molecular replacement using the coordinates of $S$. cerevisiae
Ubc7 (without U7BR) as a search model. The preliminary structure revealed helical densities on the back side of Ubc7, and U7BR from $S$. pombe was manually built into this density based on unambiguous densities for the respective side chains (Table 3).

\subsection{Comparison of ERAD E2-E2BR complexes from $H$. sapiens, S. cerevisiae and S. pombe}

$S$. pombe U7BR binds to the back side of Ubc7 as a long $\alpha$-helix comprising residues 180-213 [Figs. $1(a)$ and $1(b)$ ] in a manner that resembles E2 binding by $H$. sapiens G2BR and

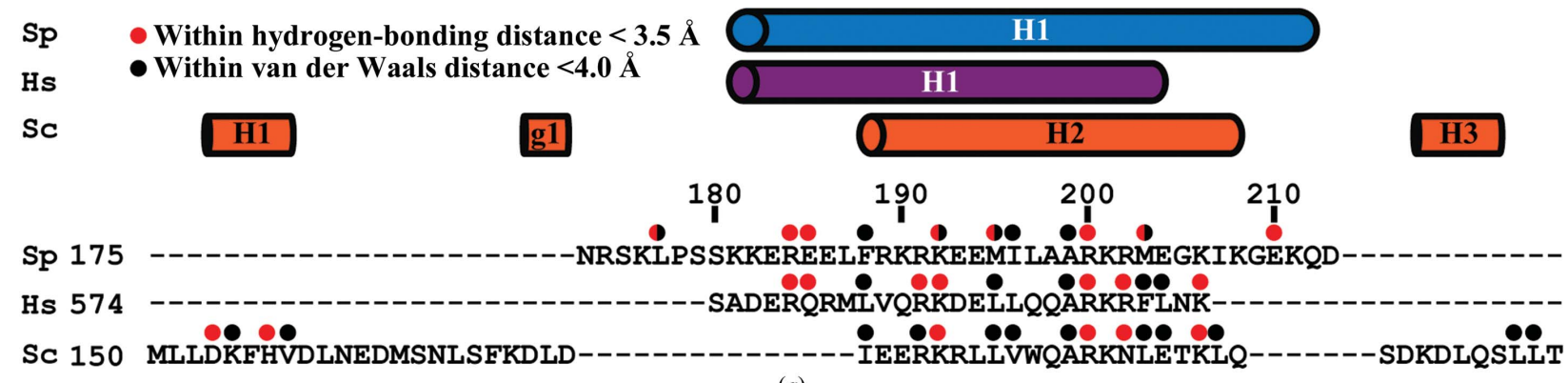

(a)
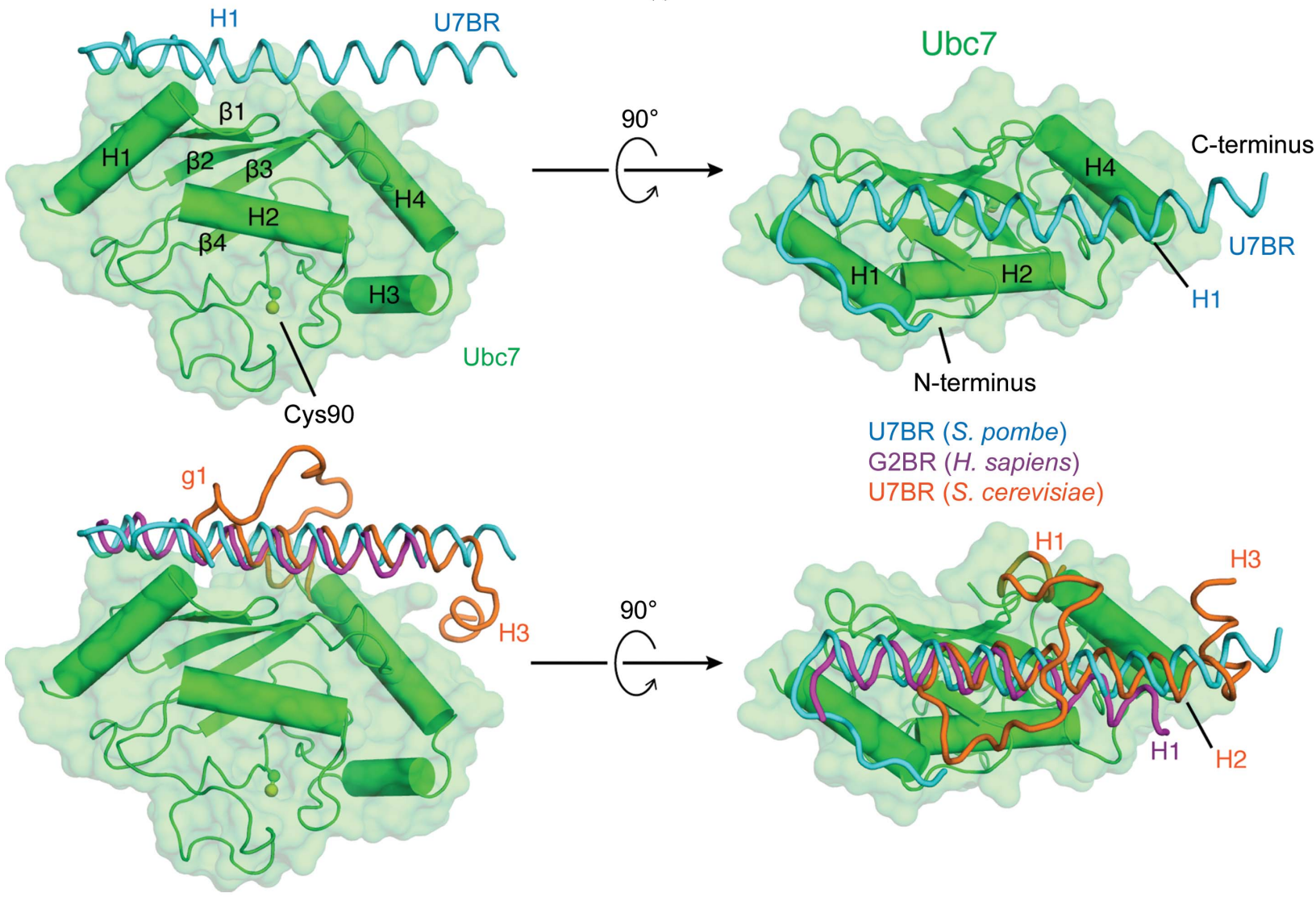

(b)

Figure 1

Crystal structure of the $S$. pombe Ubc7-U7BR complex. (a) Structure-based alignment of S. pombe U7BR, H. sapiens G2BR and S. cerevisiae U7BR. Residues within helices are represented by cylinders above the alignment. Residues that interact with Ubc7 are labeled with red or black circles. $(b)$ $S$. pombe Ubc7 shown as a green cartoon and surface with U7BR represented as a cyan ribbon. Cys 90 of Ubc7 is shown as sticks and spheres, and the Nand C-termini of U7BR are labeled. Below, similar structures of the E2BRs of H. sapiens (purple; PDB entry 3h8k; Das et al., 2009) and S. cerevisiae (orange; PDB entry 4jqu) superimposed. Figures were made with PyMOL (v.2.0; Schrödinger). 
S. cerevisiae U7BR (Li et al., 2009; Das et al., 2009; Metzger et al., 2013). The E2-binding helices (S. pombe U7BR helix H1, $H$. sapiens $\mathrm{G} 2 \mathrm{BR}$ helix $\mathrm{H} 1$ and $S$. cerevisiae U7BR helix $\mathrm{H} 2$ ) align with respect to the E2 between residues 188 and 204 of S. pombe U7BR [residues 173 and 189 of $S$. cerevisiae U7BR; residues 582 and 598 of $H$. sapiens G2BR; Fig. 1(b)]. At the

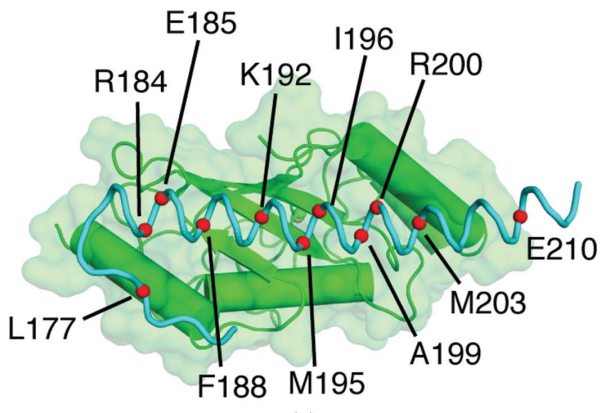

(a)

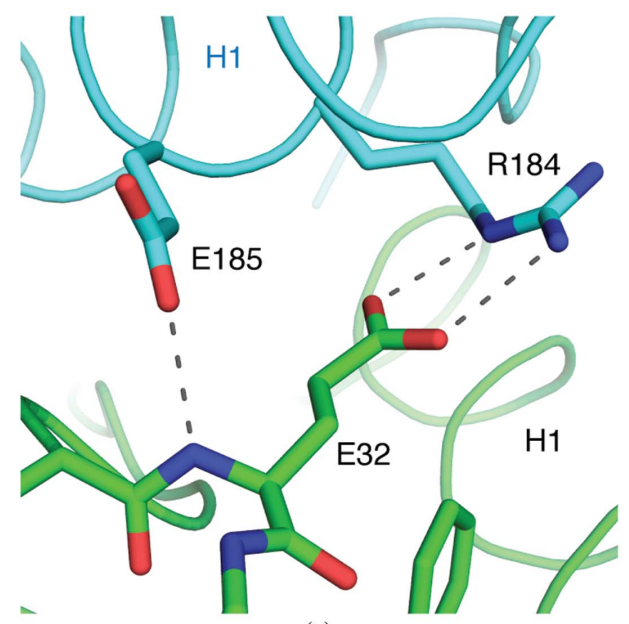

(c)

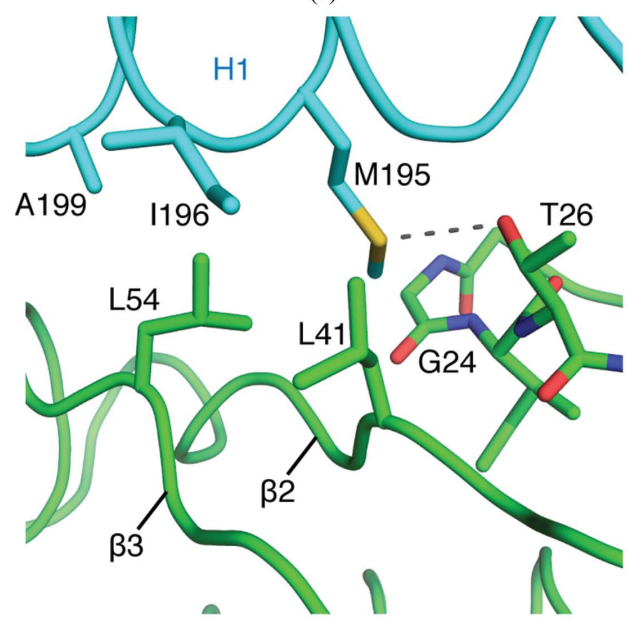

(e)



(d)

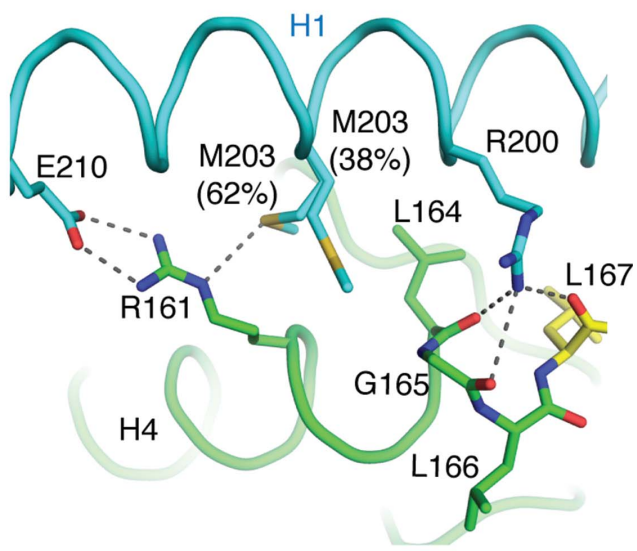

$(f)$

Figure 2

Intermolecular contacts between Ubc7 and U7BR. (a) S. pombe Ubc7-U7BR complex with $\alpha$ carbons of U7BR residues in contact with Ubc7 depicted as red spheres. (b) Interaction between Leu177 of U7BR (cyan) and Tyr14, Lys15 and Glu19 of Ubc7 (green). (c) Interaction between Arg184 and Glu185 of U7BR (cyan) and Glu32 of Ubc7 (green). (d) Interaction between Phe188 and Lys192 of U7BR (cyan) and Thr26-Ser30 and Leu41 of Ubc7 (green). (e) Interaction between Ala199, Lys192, Met195 and Ile196 of U7BR (cyan) and Gly24, Thr26, Leu41 and Leu54 of Ubc7 (green). ( $f$ ) Interaction between Arg200, Met203 and Glu210 of U7BR (cyan) and Arg161 and Leu164-Leu167 of Ubc7 (green and yellow). Residues in yellow are part of a thrombin cleavage site artifact at the C-terminus of Ubc7. 
C-terminal end of human G2BR, the remainder of helix H1 (residues 599-60) bends towards the $\beta 2 \beta 3$ loop of UBE2G2. The C-terminal ends of the two yeast U7BR helices continue to align until residue 208 of $S$. pombe U7BR (residue 193 of $S$. cerevisiae U7BR). The C-terminal end of $S$. pombe U7BR continues as a helix until residue 213, making it the longest contiguous helix of the three E2BRs. After residue 193, S. cerevisiae U7BR bends towards helix $\mathrm{H} 4$ of Ubc7, forming a separate $\alpha$-helix, H3, composed of residues 196-200. At the $\mathrm{N}$-terminal end of the helix, S. cerevisiae U7BR (residues 150172) turns towards the $\mathrm{H} 2$ face of Ubc7, forming a short $3_{10}$-helix (g1), before looping over its own E2-binding $\mathrm{H} 2$ helix to form an N-terminal $\mathrm{H} 1 \alpha$-helix. In contrast, the $\mathrm{N}$-termini of S. pombe U7BR and H. sapiens G2BR have longer E2-binding helices that extend over the $\mathrm{H} 1$ helix of the E2. Human G2BR and $S$. pombe U7BR diverge at residue 180 of $S$. pombe U7BR (residue 574 of G2BR), where human G2BR ends and S. pombe $\mathrm{U} 7 \mathrm{BR}$ turns towards the $\mathrm{H} 2$ face of $\mathrm{Ubc7}$, forming a seven-residue $\mathrm{N}$-terminal extension that lies on top of the $\mathrm{H} 1$ helix of Ubc7 and is unique among the three E2BRs. While the $H$. sapiens and S. cerevisiae E2BR domains used for crystallization were mostly ordered, with only a few residues at the $\mathrm{N}$-terminus of G2BR that did not have sufficient density to be modeled, the longer $S$. pombe U7BR construct used for crystallization had $22 \mathrm{~N}$-terminal residues that were not observed in electron density (Li et al., 2009; Das et al., 2009; Metzger et al., 2013). The $S$. pombe Ubc7-U7BR complex buries a surface area of $2423 \AA^{2}$ between the E2 and U7BR, as calculated using CNS (Brunger, 2007). For comparison, the E2-E2BR complexes from $S$. cerevisiae and $H$. sapiens bury surface areas of 2076 and $1947 \AA^{2}$ between the E2 and the E2BR, respectively.

\subsection{Ubc7-U7BR intermolecular contacts}

11 U7BR residues have main-chain or side-chain atoms within hydrogen-bonding or van der Waals distance of Ubc7 [Fig. 2(a)]. While similarities exist with respect to intermolecular contacts within the $H$. sapiens, $S$. cerevisiae and $S$. pombe E2-E2BR complexes, many of the contacts discussed here are unique to the $S$. pombe system. In the $\mathrm{N}$-terminal portion of $S$. pombe U7BR, the backbone amide $\mathrm{N}$ atom of Leu177 of U7BR is $2.8 \AA$ from a side-chain carboxylate $\mathrm{O}$ atom of Glu19 in Ubc7, to which it may establish a hydrogen bond [Fig. 2(b)]. The $\delta 1$ carbon of the Leu177 side chain is within van der Waals distance of the $\varepsilon$ and $\zeta$ carbons of Tyr14 in the H1 helix of Ubc7, and its $\delta 2$ carbon is $3.7 \AA$ from the $\alpha$ and $\beta$ carbons of Lys15 in the same helix, possibly forming hydrophobic interactions.

The N-terminal end of the H1 helix of U7BR approaches the $\beta 1 \beta 2$ loop of Ubc7. In this interface, the $\varepsilon$ and $\eta$ nitrogens of $\operatorname{Arg} 184$ are within hydrogen-bonding distance of the $\varepsilon$ oxygen of the side-chain carboxylate of Glu32 [Fig. 2(c)]. The main-chain amide $\mathrm{N}$ atom of Glu32 is $2.8 \AA$ from a side-chain carboxylate oxygen of Glu185 in U7BR, potentially creating an additional hydrogen bond.
The side chain of Phe 188 of U7BR contributes to a hydrophobic interface with Ubc7; the $\varepsilon$ and $\zeta$ carbons are 4.0 and $3.7 \AA$ from the $\alpha$ carbon of Gly 28 in the $\beta 1$ strand of Ubc7, respectively [Fig. 2(d)]. A $\delta$ carbon of Phe188 is $3.9 \AA$ from the main-chain $\alpha$ carbon of Ser30 in Ubc7; on the opposite side of the aromatic ring of Phe 188 , an $\varepsilon$ carbon is $3.4 \AA$ from the side-chain $\gamma 2$ carbon of Thr26. Nearby, the $\zeta$ nitrogen in the side chain of Lys 192 is $3.0 \AA$ from the side-chain hydroxyl O atom of Ser30. In addition, the $\alpha, \beta$ and $\gamma$ carbons of Lys 192 are $3.9 \AA$ from a side-chain $\delta$ carbon in Leu 41 in the $\beta 2$ strand of Ubc7, and the $\gamma$ carbon of Lys192 is $4.0 \AA$ from the $\gamma 2$ carbon in the side chain of Thr26 of Ubc7, possibly contributing hydrophobic contacts within the Ubc7-U7BR interface.

Additional contacts are established by Met195 and Ile196 of U7BR [Fig. 2(e)], with interactions between the side-chain $\delta$ sulfur of Met195 and the side-chain hydroxyl O atom of Thr26 in Ubc7, which are $3.1 \AA$ apart. The side-chain methyl carbon of Met195 is $3.8 \AA$ from one of the $\delta$ carbons of Leu54 in the $\beta 3$ strand of Ubc7 and $4.0 \AA$ from the $\alpha$ carbon of Gly 24 in the $\beta 1$ strand of Ubc7. The main-chain $\alpha$ carbon of Ile196 is $3.7 \AA$ from the side chain of Leu54 in Ubc7, and the side chain of Ile196 is within van der Waals distance of Leu41 and Leu54.

Leu54 in Ubc7 also appears to contribute hydrophobic contacts to the side chain of Ala199 in U7BR; the $\beta$ and $\delta 2$ carbons of Leu54 are $3.9 \AA$ from the side-chain $\beta$ carbon of Ala199 [Fig. 2(e)]. Adjacent to Ala199, one of the $\eta$ nitrogens of Arg200 is $3.1 \AA$ from the main-chain carbonyl oxygen of Leu164 and $3.4 \AA$ from the main-chain carbonyl oxygen of Gly165 at the C-terminus of Ubc7 in the H4 helix [Fig. 2(f)]. The same N atom in the side chain of Arg200 is $2.9 \AA$ from the main-chain carbonyl oxygen of Leu167, a nonphysiological contact as the E2 was expressed with a C-terminal thrombincleavable His tag and the final residue of S. pombe is Leu166.

The C-terminal end of U7BR includes Met203, a residue whose $\mathrm{S}$ atom is within hydrogen-bonding distance of the sidechain $\varepsilon$ nitrogen of Arg161 in the H4 helix of Ubc7 [Fig. 2(f)]. Met203 is also within van der Waals distance of Ubc7: the $\varepsilon$ carbon is $3.9 \AA$ from the side-chain $\delta$ carbon of Leu164 in the $\mathrm{H} 4$ helix of Ubc7. The side-chain $\beta$ carbon of Met203 is $3.8 \AA$ from the same $\delta$ carbon of Leu164. In an alternate side-chain conformation of Met203 (38\% occupancy), the side-chain methyl carbon is within van der Waals distance of the $\alpha$ carbon of Gly165 and the $\gamma$ carbon of Arg161 in Ubc7. In Glu210 of U7BR, the two side-chain carboxylate $\mathrm{O}$ atoms are 2.7 and $2.9 \AA$ from the two $\eta$ nitrogens of Arg161 in helix H4 of Ubc7, potentially forming a salt bridge.

\subsection{Interactions that are conserved between $\boldsymbol{H}$. sapiens, S. cerevisiae and S. pombe}

The Ubc7-U7BR structures from S. cerevisiae and S. pombe were aligned with the UBE2G2-G2BR structure from H. sapiens (Das et al., 2009; Metzger et al., 2013) [Figs. 1(a) and 1(b)]. Three S. pombe U7BR residues that interact with Ubc7 are structurally and genetically conserved in the two other species: Lys192, Ala199 and Arg200. In S. pombe U7BR, this 
lysine is within hydrogen-bonding distance of Ser30 in the $\beta 1 \beta 2$ loop of Ubc7. At the same E2BR position in S. cerevisiae, the side chain of Lys177 of U7BR is within hydrogenbonding distance of the side chain of Asp38 on the $\beta 2$ strand of Ubc7 [Fig. 3(a)]. Similarly, in the H. sapiens structure the side chain of Lys586 of G2BR is within hydrogen-bonding distance of the side chain of Glu38 on the $\beta 2$ strand of UBE2G2. In the position corresponding to Asp38 of S. cerevisiae Ubc7 and Glu38 of $H$. sapiens $\mathrm{UBE} 2 \mathrm{G} 2$, S. pombe Ubc7 has Asp39, but it is more than $3.5 \AA$ from the $\zeta$ nitrogen of Lys192 on U7BR. In the H. sapiens and $S$. cerevisiae structures, the $\beta 1 \beta 2$ loops of the E2s are too far from the conserved lysine to form a hydrogen bond.

Ala199 and Arg200 of S. pombe U7BR are also conserved; they correspond to Ala593 and Arg594 in H. sapiens G2BR and Ala184 and Arg185 in S. cerevisiae U7BR, respectively. Ala199 is within van der Waals distance of Leu54 on the $\beta 3$ strand of Ubc7 in the S. pombe structure. Similarly, Ala593 of G2BR is within van der Waals distance of Val53 on the $\beta 3$ strand of UBE2G2 and Ala184 is within van der Waals distance of Val53 on the $\beta 3$ strand of S. cerevisiae Ubc7 (Das et al., 2009; Metzger et al., 2013) [Fig. 3(b)]. This may represent a conserved hydrophobic interaction. The interaction between the side chain of $\operatorname{Arg} 200$ in S. pombe U7BR and main-chain carbonyl oxygens at the C-terminal end of Ubc7 may also be conserved. In $H$. sapiens, the $\varepsilon$ nitrogen and one of the $\eta$ nitrogens of Arg594 in G2BR are within hydrogen-bonding distance of the main-chain carbonyl oxygen of Leu163 in UBE2G2, and in the $S$. cerevisiae structure an $\eta$ nitrogen of Arg185 appears to interact with the carbonyl oxygens of Leu163 and Gly164 near the C-terminus of Ubc7 [Fig. 3(b)].

\subsection{E1-Ubc7 transthiolation in S. pombe}

S. pombe U7BR decreases the rate of Ubc7 Ub charging in vitro, similar to the effect of G2BR on UBE2G2 Ub charging in H. sapiens (Fig. 4; Das et al., 2009). Superposition of E2E2BR crystal structures on the structure of a cross-linked Ub E1-E2 complex reveals potential steric clashes between the $\mathrm{N}$-termini of $H$. sapiens $\mathrm{G} 2 \mathrm{BR}$ or $S$. pombe U7BR and the Ubfold domain (UFD) of the Ub E1 (Olsen \& Lima, 2013). In contrast, because the Ubc7-binding $\mathrm{H} 2$ helix of $S$. cerevisiae U7BR does not extend as far in the N-terminal direction, it does not cover the E2 surface contacted by the UFD. This may explain why $S$. cerevisiae U7BR is able to stimulate
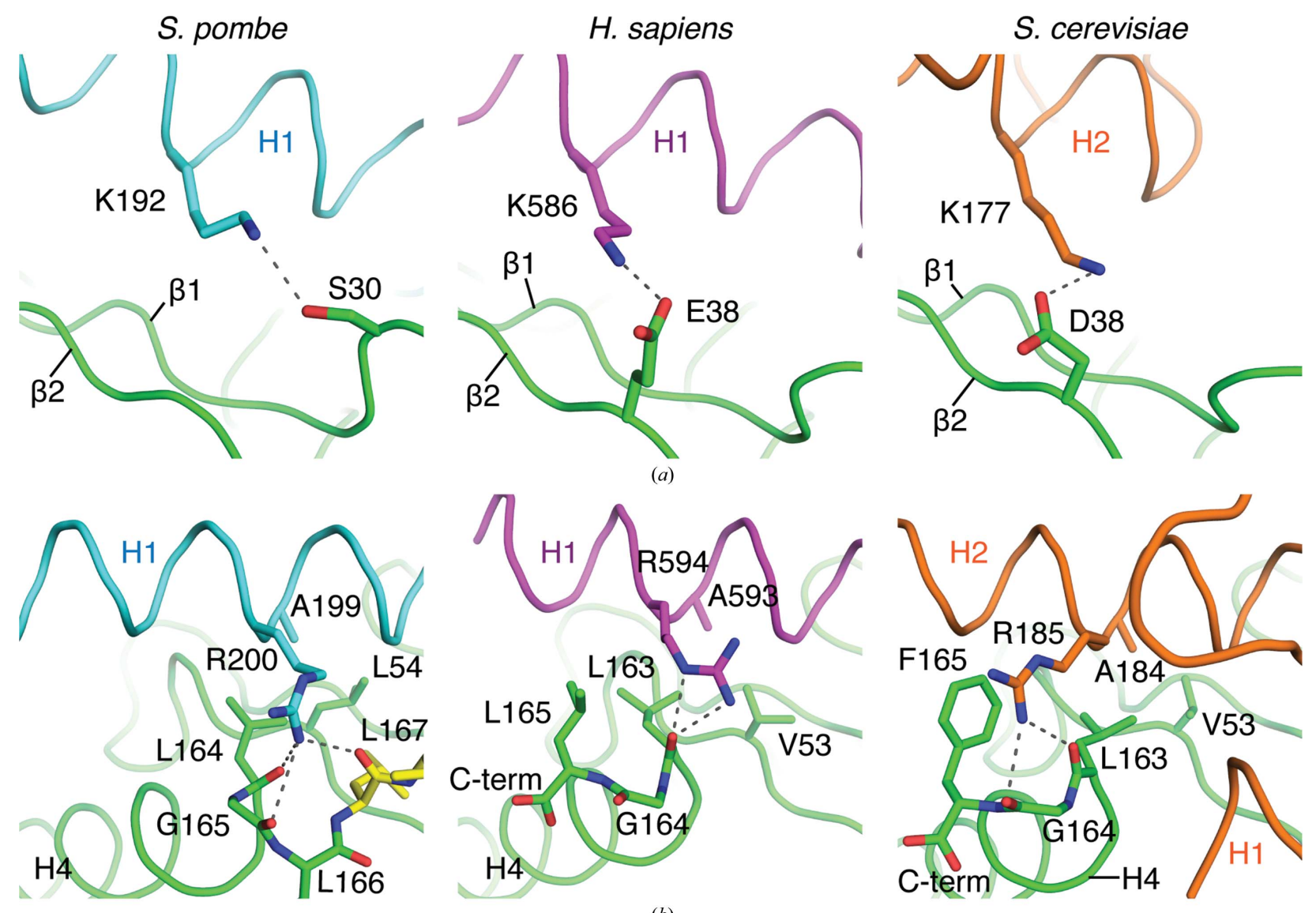

Figure 3

Conserved E2-interacting residues among ERAD E2BRs. (a) A conserved E2BR lysine may form a hydrogen bond to a Ubc7 side chain in $S$. pombe (cyan), H. sapiens (purple) and S. cerevisiae (orange). (b) A conserved alanine and arginine interact with residues of Ubc7 in S. pombe (cyan), H. sapiens (purple) and $S$. cerevisiae (orange). 


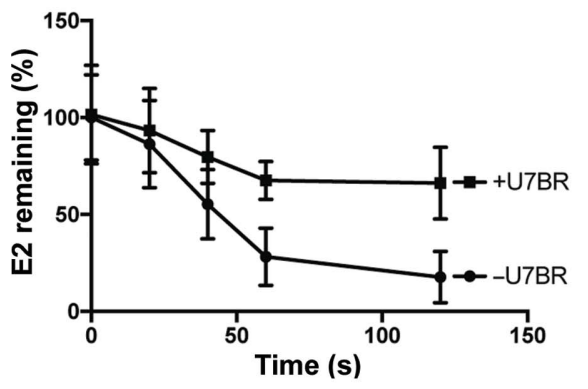

$(a)$

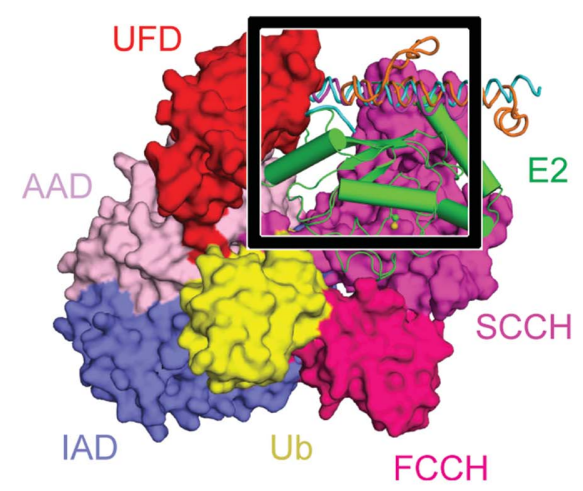

(b)

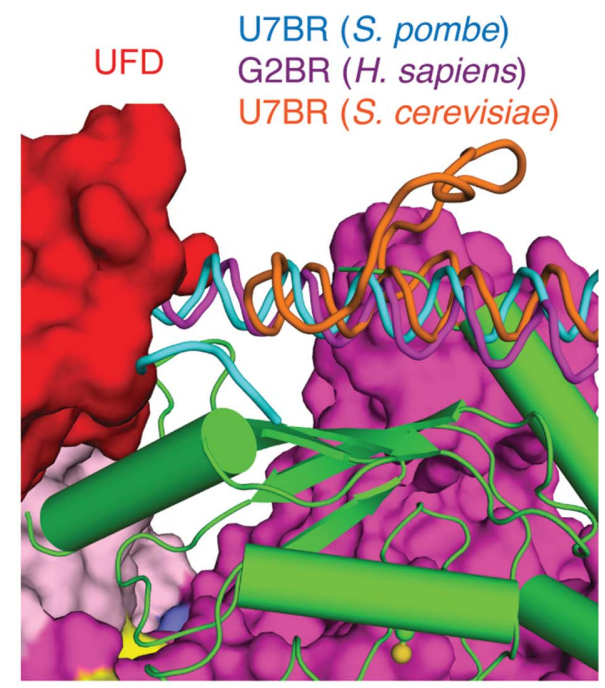

(c)

Figure 4

S. pombe U7BR impedes Ubc7 transthiolation. (a) Percentage of free Ubc7 remaining in a transthiolation assay with and without excess U7BR. Error bars represent one standard deviation. (b) E2-E2BR co-crystal structures from S. pombe (PDB entry 6op8), H. sapiens (PDB entry 3h8k) and $S$. cerevisiae (PDB entry 4jqu; Metzger et al., 2013) were modeled into the crystal structure of $S$. pombe E1-Ubc4-Ub-ATP (PDB entry 4ii2; Olsen \& Lima, 2013) by superposing the E2 structures. The black square indicates the area displayed in $(c)$. (c) The N-terminal portions of $S$. pombe U7BR and $H$. sapiens G2BR, but not $S$. cerevisiae U7BR, clash with the UFD domain of E1. IAD, inactive adenylation domain; AAD, active adenylation domain; Ub, ubiquitin; UFD, ubiquitin-fold domain; FCCH, first catalytic cysteine half-domain; SCCH, second catalytic cysteine half-domain.

transthiolation in vitro, rather than repressing it as in the other two species (Metzger et al., 2013).

While $S$. pombe U7BR appears to interfere with E1-Ubc7 binding, it presents no obvious structural impediment to Ubc7-E3 RING domain binding. When the E2 proteins from the structures of $S$. pombe Ubc7-U7BR and human UBE2G2 bound to the RING and G2BR domains of gp78 were superposed, U7BR did not preclude interactions with the RING domain-binding surface of the E2 (Das et al., 2013). This is consistent with the observation that E2BRs from both $H$. sapiens and $S$. cerevisiae enhance the E3-mediated ubiquitylation activity and RING domain-binding affinity of ERAD-associated E2s (Das et al., 2009; Metzger et al., 2013).

\section{Discussion}

Recruitment of a soluble E2 (Ubc7 in yeast and UBE2G2 in humans) to the endoplasmic reticulum is essential for many ubiquitylation events in ERAD (Zattas \& Hochstrasser, 2015). Although this core function is conserved, it is interesting that the E2-binding regions (E2BRs) have such a varied sequence and structure. In addition, isolated U7BR from $S$. cerevisiae increases the rate of E1-E2 transthiolation, suggesting that Cue1 may stimulate transthiolation at the ER (Metzger et al., 2013). In contrast, the ERAD E2BRs from $H$. sapiens and $S$. pombe appear to have the opposite effect: the E2BR inhibits E1-mediated E2 charging [Fig. 4(a) and Supplementary Fig. S1] (Das et al., 2009). This observation suggests that E2s are charged with Ub prior to ER recruitment. A structural basis for the opposing effects observed for E1-E2 transthiolation can be rationalized by superposing E2E2BR structures onto Ub E1-E2 complexes. These models reveal that the N-terminal ends of E2BRs from human and
S. pombe present a steric clash with the E2-binding Ub-fold domain (UFD) of E1 [Figs. 4(b) and 4(c)]. In contrast, the $S$. cerevisiae U7BR does not occlude the E2-binding surface of the UFD. $S$. pombe appears to be more similar to mammals than to $S$. cerevisiae with respect to the regulation of heatshock factors and some aspects of lipid metabolism; it is possible that the recruitment of soluble ERAD E2s to the ER may be another process in which $S$. pombe is a closer unicellular model organism than $S$. cerevisiae (Raychaudhuri et al., 2012; Gallo et al., 1991; Hoffman et al., 2015).

This work identified the $S$. pombe $\mathrm{U} 7 \mathrm{BR}$ and presents an atomic resolution model of a Ubc7-U7BR complex. While the E2-binding mode of $S$. pombe U7BR is globally similar to those observed in the human and $S$. cerevisiae systems, their biochemical effects and interactions include elements that are unique among the three ERAD E2-E2BR structures solved to date (Metzger et al., 2013; Das et al., 2009, 2013). The similarities and differences in E2-E2BR complexes solved thus far may aid in the identification of additional E2BRs in other systems.

\section{Acknowledgements}

The content is solely the responsibility of the authors and does not represent the official views of the National Institutes of Health. CDL is an investigator of the Howard Hughes Medical Institute. The work reported here was also based in part upon research conducted at NE-CAT beamlines (P41 GM103403, NIH NIGMS, S10 RR029205, NIH-ORIP HEI grant). The beamline research used resources of the Advanced Photon Source, a US Department of Energy (DOE) Office of Science User Facility operated for the DOE Office of Science by Argonne National Laboratory under Contract No. DE-AC02- 
06CH11357. Author contributions were as follows. Sequence alignments and identification of Cue4 were performed by MBM and AMW. Cloning, purification, crystallization, structure determination and biochemical experiments were performed by ZSH and CDL. Data were analyzed and the manuscript was prepared by ZSH and CDL, with comments provided by MBM and AMW. The authors declare no competing financial interests.

\section{Funding information}

This research was supported in part by the National Institute of General Medical Sciences of the National Institutes of Health under award Nos. R01GM065872 (CDL), R35GM118080 (CDL) and P30 CA008748 (NIH NCI-Cancer Center Support Grant) and the Intramural Research Program of the National Institutes of Health, National Cancer Institute, Center for Cancer Research (MBM and AMW).

\section{References}

Adams, P. D., Afonine, P. V., Bunkóczi, G., Chen, V. B., Davis, I. W., Echols, N., Headd, J. J., Hung, L.-W., Kapral, G. J., GrosseKunstleve, R. W., McCoy, A. J., Moriarty, N. W., Oeffner, R., Read, R. J., Richardson, D. C., Richardson, J. S., Terwilliger, T. C. \& Zwart, P. H. (2010). Acta Cryst. D66, 213-221.

Bazirgan, O. A. \& Hampton, R. Y. (2008). J. Biol. Chem. 283, $12797-$ 12810.

Biederer, T., Volkwein, C. \& Sommer, T. (1996). EMBO J. 15, 2069 2076.

Biederer, T., Volkwein, C. \& Sommer, T. (1997). Science, 278, $1806-$ 1809.

Brunger, A. T. (2007). Nature Protoc. 2, 2728-2733.

Chen, B., Mariano, J., Tsai, Y. C., Chan, A. H., Cohen, M. \& Weissman, A. M. (2006). Proc. Natl Acad. Sci. USA, 103, 341-346.
Das, R., Liang, Y.-H., Mariano, J., Li, J., Huang, T., King, A., Tarasov, S. G., Weissman, A. M., Ji, X. \& Byrd, R. A. (2013). EMBO J. 32, 2504-2516.

Das, R., Mariano, J., Tsai, Y. C., Kalathur, R. C., Kostova, Z., Li, J., Tarasov, S. G., McFeeters, R. L., Altieri, A. S., Ji, X., Byrd, R. A. \& Weissman, A. M. (2009). Mol. Cell, 34, 674-685.

Emsley, P., Lohkamp, B., Scott, W. G. \& Cowtan, K. (2010). Acta Cryst. D66, 486-501.

Gallo, G. J., Schuetz, T. J. \& Kingston, R. E. (1991). Mol. Cell. Biol. 11, 281-288.

Guerriero, C. J. \& Brodsky, J. L. (2012). Physiol. Rev. 92, 537-576.

Hiller, M. M., Finger, A., Schweiger, M. \& Wolf, D. H. (1996). Science, 273, 1725-1728.

Hoffman, C. S., Wood, V. \& Fantes, P. A. (2015). Genetics, 201, $403-$ 423.

Kostova, Z., Mariano, J., Scholz, S., Koenig, C. \& Weissman, A. M. (2009). J. Cell Sci. 122, 1374-1381.

Li, W., Tu, D., Li, L., Wollert, T., Ghirlando, R., Brunger, A. T. \& Ye, Y. (2009). Proc. Natl Acad. Sci. USA, 106, 3722-3727.

McCoy, A. J., Grosse-Kunstleve, R. W., Adams, P. D., Winn, M. D., Storoni, L. C. \& Read, R. J. (2007). J. Appl. Cryst. 40, 658-674.

Metzger, M. B., Liang, Y.-H., Das, R., Mariano, J., Li, S., Li, J., Kostova, Z., Byrd, R. A., Ji, X. \& Weissman, A. M. (2013). Mol. Cell, 50, 516-527.

Olsen, S. K. \& Lima, C. D. (2013). Mol. Cell, 49, 884-896.

Otwinowski, Z. \& Minor, W. (1997). Methods Enzymol. 276, 307-326.

Prag, G., Misra, S., Jones, E. A., Ghirlando, R., Davies, B. A., Horazdovsky, B. F. \& Hurley, J. H. (2003). Cell, 113, 609-620.

Ravid, T., Kreft, S. G. \& Hochstrasser, M. (2006). EMBO J. 25, $533-$ 543.

Raychaudhuri, S., Young, B. P., Espenshade, P. J. \& Loewen, C. (2012). Curr. Opin. Cell Biol. 24, 502-508.

Smith, M. H., Ploegh, H. L. \& Weissman, J. S. (2011). Science, 334, 1086-1090.

Swanson, R., Locher, M. \& Hochstrasser, M. (2001). Genes Dev. 15, 2660-2674.

Tiwari, S. \& Weissman, A. M. (2001). J. Biol. Chem. 276, 16193-16200.

Ye, Y. \& Rape, M. (2009). Nature Rev. Mol. Cell Biol. 10, 755-764.

Zattas, D. \& Hochstrasser, M. (2015). Crit. Rev. Biochem. Mol. Biol. 50, 1-17. 